\title{
Standing up against workplace bullying behavior: Recommendations from newly licensed nurses
}

\author{
Carolyn R. Smith*1, Steven J. Palazzo², Paula L. Grubb ${ }^{3}$, Gordon L. Gillespie ${ }^{1}$ \\ ${ }^{1}$ College of Nursing, University of Cincinnati, Cincinnati, USA \\ ${ }^{2}$ College of Nursing, Seattle University, Seattle, USA \\ ${ }^{3}$ National Institute for Occupational Safety and Health, Centers for Disease Control and Prevention, Cincinnati, USA
}

Received: March 9, 2020

DOI: $10.5430 /$ jnep.v10n7p35
Accepted: April 7, 2020

Online Published: April 12, 2020

\begin{abstract}
Objective: Workplace bullying exists in today's healthcare system and often targets newly licensed nurses. Experiences of workplace bullying behavior may negatively affect the nurses' physical and psychological health and impact job satisfaction and staff turnover rates at an organizational level. The purpose of this study was to explore strategies suggested by newly licensed nurses to prevent and intervene during incidents of workplace bullying behavior.

Methods: An exploratory qualitative design guided this study. Three open-ended questions asked included: What do you think could be done to prevent a future, similar incident of workplace bullying? If you or someone else attempted to the stop the bullying incident, please describe the actions taken. If you or someone else did not attempt to stop the bullying incident, please state what would need to happen for you to intervene on behalf of yourself or someone else. Surveys were distributed electronically to newly licensed nurses from three baccalaureate nursing programs who had participated in a workplace bullying education intervention study as students. A total of 79 responses were received. Responses to three open-ended questions about recent incidents of workplace bullying behavior were coded and analyzed. Then the Social-Ecological Model was used to organize results into individual, relationship, and organizational level strategies.

Results: Most respondents reported experiencing workplace bullying behaviors in the previous six months. Three domains of strategies were identified: Preventing Future Bullying Behavior, Stopping Incidents of Bullying Behavior, and Promoting Others to Act.

Conclusions: Results indicated newly licensed nurses desire to be supported by their peers and organization as well as strategies to intervene when bullying behaviors occur. Implications for clinical practice and education are presented.
\end{abstract}

Key Words: Workplace bullying, Workplace aggression, Workplace violence, Newly licensed nurse

\section{INTRODUCTION}

Workplace bullying (WB) in healthcare remains a persistent problem, particularly against newly licensed nurses. ${ }^{[1,2]}$ Among healthcare workers, frequently experienced WB behaviors included being assigned an unmanageable workload $(62 \%, \mathrm{n}=149)$, being ignored by others $(35 \%, \mathrm{n}=85)$, coworkers withholding information need for clinical care $(28 \%, \mathrm{n}=68)$, and being humiliated or ridiculed $(22 \%, \mathrm{n}$ $=54) \cdot{ }^{[3]}$ In nurses, negative outcomes of WB behaviors include burnout and lack of engagement, ${ }^{[4]}$ decreased work productivity, ${ }^{[2]}$ organizational mistrust, ${ }^{[5]}$ turnover intention, ${ }^{[6]}$ sleep difficulties, ${ }^{[7]}$ and general physical and mental health

*Correspondence: Carolyn R. Smith; Email: Carolyn.Smith@uc.edu; Address: P.O. Box 210038, Cincinnati, OH 45221-0038, USA. 
complaints. ${ }^{[1,2,7]}$

Debate still exists surrounding what label best reflects bullying behaviors experienced by nurses in the workplace (e.g., lateral violence, horizontal violence, mobbing). ${ }^{[8]}$ Despite the debate, most researchers agree that bullying in nursing workplaces consist of "repeated, cumulative, and patterned form of negative behaviors of a perpetrator abusing his or her power over time toward the victim, resulting in the profound negative impact on the bully victim and organization" ${ }^{[9]}$ (p. 263).

Several strategies have been recommended to address the persistence of WB in healthcare. First, the work culture needs to be redesigned to foster collegiality and a violence free workplace. Several researchers encourage the adoption of a "no tolerance policy". ${ }^{[10-12]}$ While this policy is a good start towards creating a safe work culture, the implementation of such a policy is typically not described, does not account for one-time behaviors atypical for the offender, and focused on how to respond after the event has already occurred. This necessitates the need for further interventions to be considered which are prevention-focused. Castronovo, Pullizzi, and Evans (2016) recommend hospitals adopt an assessment and public reporting process related to $\mathrm{WB}$ with the assumption hospitals will more effectively focus on prevention to prevent negative findings from being reported to the public. ${ }^{[13]}$ Sguera et al. (2016) recommend team building sessions as a viable strategy to combat WB. ${ }^{[6]}$ Team building in addition to other WB interventions requires all employees, staff and administration, to participate in work culture changes. ${ }^{[11]}$ These changes coupled with strong diversity climates may lead to a reduction of WB behaviors. Diversity climates refer to efforts by organizations to actively mitigate status differences $^{[4]}$ and target gender, ethnicity, sexual-orientation, and role. Sliter et al. (2014) reported increased worker engagement and acceptance of others in work environments with strong diversity climates. ${ }^{[4]}$

Developing a positive work culture requires effective communication between employees. Effective communication is believed to reduce the incidence of $\mathrm{WB} .{ }^{[14]}$ It is important employees receive education on how to confront other employees demonstrating WB behaviors. ${ }^{[15]}$ In a recent review of the literature, Koh (2016) recommends cognitive rehearsal as one strategy in developing effective communication among employees; concluding cognitive rehearsal when used with scripting specific responses in the presence of WB can help mitigate this behavior. ${ }^{[14]}$ Ceravolo et al. (2012) delivered a workshop series on WB which emphasized interpersonal communications finding their intervention decreased the incidence of WB from $90 \%$ to $76 \%$. $^{[10]}$
Interventions such as communication workshops to combat WB require institutional resources and front-line managers as well as hospital administrators to oversee the use of these resources. This oversight relies on the leadership team being instrumental in identifying WB and creating safe work environments. ${ }^{[10,16]}$ For effective leadership, individuals in these positions need to role model positive behaviors in the workplace. ${ }^{[17]}$ This role modeling can be demonstrated by addressing incidents of WB as they occur even when the aggressor may be a respected employee. ${ }^{[15]}$ In some organizations, the effectiveness of administrators and thus the organization are perceived as poor. This negative interpretation is commonly the result of some employees perceiving that the organization provides greater support to the aggressor than the target. ${ }^{[5]}$ As a result, further research is needed to better understand WB in relation to the need for a safe work culture, effective communication, and improved administrator effectiveness. While most research is focused on describing the problem of WB against nurses, only some research has been trialed to test interventions. Greater depth is needed to identify additional strategies to prevent and mitigate WB.

One way to examine and categorize strategies to address WB is within the context of the Social-Ecological Model. ${ }^{[18,19]}$ The Social-Ecological Model proposes there are four levels which can influence violence: individual, relationship, community, and societal. According to the model, the individual level examines factors such as a person's biological and personal history that may influence risk of being a victim or perpetrator. Intervention strategies at this level need to target individuals' attitudes, beliefs, and behaviors. The relationship level examines interpersonal relationships that may influence an individual's behavior and, as a result, contribute to them being a victim or perpetrator of violence. Proposed interventions at this level should aim to promote healthy interpersonal relationships through strategies such as conflictresolution skills, effective communication, and mentoring. The community level looks at the context where social relationships occur such as workplaces, schools, communities, etc. Violence interventions geared toward this level include context specific policies, resources, and environments to support healthy relationships. The final level, societal, explores how societal and cultural norms, policies, and resources influence violence. Interventions at this level target policies and inequity that may promote victimization and perpetration of violence. The purpose of this study was to explore strategies recommended by newly-graduated nurses exposed to WB behaviors. 


\section{Methods}

An exploratory qualitative design was used for this study. This research was part of a larger study exploring workplace bullying behaviors against nursing students and newlylicensed registered nurses. Institutional Review Board approvals were obtained from all study sites prior to participant enrollment and data collection.

\subsection{Settings and sample}

In Fall 2013, junior and senior-level students enrolled in three university-based baccalaureate of nursing programs in the Midwestern United States were invited to participate in a larger study addressing WB. Of the 335 eligible students, 185 (55.2\%) provided informed consent and enrolled. The students received an educational intervention focused on increasing their knowledge of and ability to recognize behaviors that are WB. ${ }^{[20]}$ Thirty months after initial study enrollment, the junior nursing students had been registered nurses for less than one year and the senior nursing students less than 2 years.

\subsection{Definition for WB behaviors}

From January through April 2014, study participants received an educational intervention focused on exploring WB. WB was defined as intense, negative behaviors intended to cause the victim to feel threatened, vulnerable, and powerless and which persist for a minimum of least six months. Considering all the current study respondents received the same educational intervention, the authors assumed respondents would recall and apply this definition of WB when answering the current survey questions.

\subsection{Current procedures}

During March and April 2016, all 185 study participants were emailed the current study survey link via SurveyMonkey (San Mateo, CA), a web-based HIPAA compliant survey collector. Participants who had experienced an incident of bullying behavior in the previous six months were asked to respond to three open-ended questions:

1) What do you think could be done to prevent a future similar incident? This question was asked to facilitate participant use of a systems' focus to identify prevention strategies of actual versus hypothetical events.

2) If you or someone else attempted to the stop the incident of bullying behavior, please describe the actions that were taken to stop the action. This question aimed to identify a pool of strategies used in practice settings that may be novel in relation to recommendations made by workplace bullying experts.

3) If you or someone else did not attempt to stop the incident of bullying behavior, please state what would need to happen

Published by Sciedu Press for you to intervene on behalf of yourself or someone else to stop the incident. This question was asked because in nursing we need leaders at the bedside who will intervene on behalf of themselves or others when workplace bullying behavior occurs. Determining the circumstances, changes, interventions, etc., from the study participants to promote this leadership at the bedside is crucial.

Participants typed responses to questions directly into the web-based collector. Researchers transferred responses into a Microsoft Word (Redmond, WA) document for analysis.

\subsection{Data analysis and trustworthiness}

The qualitative data were analyzed using a descriptive content analysis approach. ${ }^{[21,22]}$ First, the researchers read the data several times to obtain an essence of their composite meaning. Second, the researchers identified phrases mentioned by several participants which were deemed to answer the three research questions. Third, the researchers met and discussed each phrase and made decisions by consensus whether to keep or disregard. Fourth, the researchers developed a coding schema clustering the phrases by domain (i.e. which research question it answered) and Social-Ecological Model level (i.e. individual, relationship, community, societal). Fifth, the researchers independently coded each line of data according to the coding schema. Sixth, the researchers met and discussed their coding line by line. When discrepancies occurred in coding with at least one researcher, the researchers provided rationale for their coding decision. Discussion ensued until consensus was achieved for the coding of each phrase. Seventh, the researchers used NVivo 10 (Burlington, MA) to manage the phrases within their respective domains. Eighth, the researchers critiqued the NVivo output files to confirm the phrases were assigned to the correct domains. No errors were noted. No recommendations were provided to change the names of the domains or domain descriptions.

Team members endeavored to ensure the trustworthiness of study results through credibility, transferability, dependability, and confirmability strategies. ${ }^{[23]}$ Credibility was enriched by providing representative statements for each domain of the study results and triangulation of sources. The study participants represented a diverse sample of newly licensed nurses from multiple practice settings. This heterogeneity of the sample allowed for a triangulation of sources (participants). Transferability was enriched by authors providing context for the study findings, so readers can make determinations about the applicability of the study findings to their respective work settings. Dependability was improved through an external audit. While three researchers on the study team performed the qualitative analysis, the fourth re- 
searcher conducted an external audit. During the audit, the fourth researcher examined the coding schema, coding decisions, and representative statements and judged the accuracy of the study findings based on the study procedures and data. Confirmability was increased by having three researchers first independently analyze the data and then come to consensus on the coding schema and subsequent representative domains, subdomains, and quotes. Notes were maintained for these coding decisions.

\section{Results}

Of the 185 study participants, $79(42.7 \%)$ submitted responses to the current survey. Sixty-eight $(86 \%)$ selfidentified as female, $67(84 \%)$ self-reported their race as
Caucasian, and $62(78.5 \%)$ reported being employed fulltime as a registered nurse.

As shown in Table 1 , over $75 \%(n=61)$ of participants selfreported experiencing bullying behaviors in the previous six months and were currently employed in a variety of nursing settings. Aggressors identified included fellow nurses, physicians, charge nurses, and other hospital staff. Less than half $(n=29)$ of those who experienced bullying behaviors had someone intervene, help mitigate, or stop the bullying incident. Interveners included themselves, fellow nurses, administrator/supervisors, and others. Table 1 contains descriptive statistics about the work setting of participants, aggressors, and interveners.

Table 1. Target's Department of Employment and Types of Aggressors and Interveners

\begin{tabular}{|c|c|}
\hline Variable & Number (\%) \\
\hline \multicolumn{2}{|l|}{ Departments of Employment $(n=61)$} \\
\hline Intensive Care or Critical Care Unit & $26(42.6 \%)$ \\
\hline Non-Intensive Care Unit (e.g., med/surg, orthopedic, neurological) & $8(13.1 \%)$ \\
\hline Emergency/Trauma & $6(9.8 \%)$ \\
\hline Public Health Setting (e.g., school, rehab, long-term care) & $5(8.2 \%)$ \\
\hline Hematology/Oncology & $4(6.6 \%)$ \\
\hline Pediatrics & $4(6.6 \%)$ \\
\hline Obstetrics/Labor \& Delivery & $2(3.3 \%)$ \\
\hline Inpatient Float Pool & $2(3.3 \%)$ \\
\hline Psychiatric/Mental Health & $1(1.6 \%)$ \\
\hline Did not report & $3(4.9 \%)$ \\
\hline \multicolumn{2}{|l|}{ Aggressors $(n=60)$} \\
\hline Registered Nurse & $31(39.2 \%)$ \\
\hline Preceptor, Charge Nurse, Supervisor, Administrator & $13(16.5 \%)$ \\
\hline Physician & $10(12.7 \%)$ \\
\hline Other healthcare worker & $6(7.6 \%)$ \\
\hline \multicolumn{2}{|l|}{ Interveners $(n=29)$} \\
\hline Self & $17(58.6 \%)$ \\
\hline Preceptor, Charge Nurse, Supervisor, Administrator & $8(27.6 \%)$ \\
\hline $\mathrm{RN}$ & $3(10.3 \%)$ \\
\hline Other healthcare worker & $1(3.5 \%)$ \\
\hline
\end{tabular}

Phrases from responses to the three open-ended questions were organized into domains representing strategies to address workplace bullying behavior. Strategies fell into one of three domains: Preventing Future Bullying Behavior, Stopping Incidents of Bullying Behavior, and Promoting Others to Act. Within each domain, phrases were interpreted through the lens of the Social-Ecological Model ${ }^{[18,19]}$ to further sort strategies into one of three levels: individual, relationship, and community. Individual-level strategies were ones connected to an individual's beliefs, attitudes, and be- haviors. Relationship-level strategies focused on addressing the ways individuals relate to and communicate with one other. Community-level strategies targeted factors found within and controlled by the workplace community or organization (e.g., overall climate, system structure, policies and procedures). No participant responses reported strategies which fit the societal level of the model. Descriptions of each domain and corresponding strategies as organized by levels of the Social-Ecological Model are presented below as well as summarized in Table 2. 
Table 2. Suggested strategies by domain and level of Social-Ecological Model

\begin{tabular}{|c|c|c|c|}
\hline Domain & Individual level & Relationship level & Organizational level \\
\hline $\begin{array}{l}\text { Preventing } \\
\text { Future } \\
\text { Bullying } \\
\text { Behavior }\end{array}$ & $\begin{array}{l}\text { - Taking a communication } \\
\text { class } \\
\text { - Expressing confidence in } \\
\text { my skills } \\
\text { - Adopting an open-minded } \\
\text { attitude }\end{array}$ & $\begin{array}{l}\text { - Helping coworkers } \\
\text { o Ask others for help } \\
\text { o Interrupt bullying incident and } \\
\text { give each nurse a chance to speak } \\
\text { o Remind others about need for } \\
\text { professional, respectful } \\
\text { exchanges } \\
\text { o Talk with staff nurses about } \\
\text { stressors and attitudes } \\
\text { o Address nurses known for bullying } \\
\text { behaviors } \\
\text { - Addressing bully after event } \\
\text { o Confront the bully } \\
\text { o Communicate how behavior } \\
\text { makes one uncomfortable } \\
\text { o Speak directly to the aggressor } \\
\text { instead of going to HR }\end{array}$ & $\begin{array}{l}\text { - Fostering positive, team-oriented work } \\
\text { environment } \\
\text { o Team-focused work environment } \\
\text { o Supportive culture } \\
\text { o Increase presence on unit } \\
\text { - Using resources to prevent workplace } \\
\text { bullying behavior } \\
\text { o Orient preceptors to new nurses' } \\
\text { abilities } \\
\text { o Mentorship programs } \\
\text { o Education on effective and } \\
\text { professional communication } \\
\text { o Zero tolerance policy for bullying } \\
\text { behaviors } \\
\text { o Investigate the issue } \\
\text { o Better staffing }\end{array}$ \\
\hline $\begin{array}{l}\text { Stopping } \\
\text { Incidents of } \\
\text { Bullying } \\
\text { Behavior }\end{array}$ & $\begin{array}{l}\text { - Understanding with clarity } \\
\text { the scope of practice and } \\
\text { job description }\end{array}$ & $\begin{array}{l}\text { - Discussing incident and/or seek } \\
\text { support from peers/manager } \\
\text { - Using de-escalation strategies } \\
\text { o Try to get on same page } \\
\text { o Adopt appropriate tone of voice } \\
\text { o Remind aggressor that you are } \\
\text { still learning } \\
\text { o Try to defuse situation } \\
\text { - Upstanding activities } \\
\text { o Step in and mediate incident } \\
\text { o Encourage aggressor to } \\
\text { understand others' perspective } \\
\text { - Responding with aggression } \\
\text { o Talk over aggressor to redirect } \\
\text { conversation } \\
\text { o Call out bullying behaviors as } \\
\text { unacceptable }\end{array}$ & $\begin{array}{l}\text { - Re-establishing equilibrium } \\
\text { o Keep bully away from others } \\
\text { o Change in patient assignment } \\
\text { o Remove or fire bully from unit } \\
\text { - Engaging the system } \\
\text { o Report the aggressor and incident } \\
\text { using workplace system } \\
\text { o Involve Human Resources and } \\
\text { Management } \\
\text { o Investigate bullying behavior } \\
\text { incident }\end{array}$ \\
\hline $\begin{array}{l}\text { Promoting } \\
\text { Others to } \\
\text { Act }\end{array}$ & - None & $\begin{array}{l}\text { - Fostering relationships in the } \\
\text { workplace } \\
\text { o Get to know coworkers better so } \\
\text { you can identify when they are } \\
\text { escalating } \\
\text { o Understand the role of other } \\
\text { professions } \\
\text { o Better communication between } \\
\text { professions }\end{array}$ & $\begin{array}{l}\text { - Creating a safe work culture } \\
\text { o Safe culture for confronting WPB } \\
\text { o Create policy about workplace } \\
\text { behavior } \\
\text { o Supportive and respectful work } \\
\text { environment } \\
\text { o Reporting will lead to change } \\
\text { o Have outlets for work stress } \\
\text { - Preventing bullying behaviors through } \\
\text { administrative support and resources } \\
\text { o Increased staffing } \\
\text { o Have another level of command } \\
\text { between management and staff } \\
\text { o Reporting system for bullying } \\
\text { behaviors }\end{array}$ \\
\hline
\end{tabular}




\subsection{Preventing future bullying behavior}

Participants were asked what could be done to prevent future incidents of WB. Three strategies were identified at the individual level and focused on addressing an individual's attitudes, beliefs, and knowledge. Several participants believed bullying occurred due to communication breakdowns. One participant summed up this sentiment in the following quote: "You often find there are often misunderstandings that can be that can easily be worked out through [nurses using] effective communication." Therefore, the first strategy identified was Taking a communication class. This strategy was specifically mentioned in the following participant remark: "Tell the staff nurse [who bullied] to take a communication class." Nurses believed increasing knowledge of effective communication skills by taking a class could reduce future WB incidents. Another individual level strategy recommended was Expressing confidence in my skills. Having a belief in oneself and projecting that confidence to others were thought as a way one could deter future WB. Upon reflection of how to prevent another incident of WB, one participant remarked "I could have spoken up about it [WB incident]." Similarly, another respondent stated "The number of years [on the job] don't necessarily mean anything... I feel confident in my potential regardless." Finally Adopting an open-minded attitude about others' perspectives was suggested as a WB prevention strategy. One participant stated "To be honest, I'm not sure [what can be done to prevent WB in future]. This job is going to have stress and people can respond negatively to stress. I guess... have open minds to other's concerns [can help]."

Numerous responses to prevent future WB represented strategies focused on the relationship-level or addressing one's relationship to and with others. The first strategy was Helping coworkers and consisted of ideas focused on seeking out and/or providing help to coworkers to prevent future WB. Participants discussed asking [coworkers] for help after a WB event as well as asking coworkers to help address nurses known for WB behaviors. Helping coworkers also included wanting coworkers to step in and interrupt future WB incidents so that each nurse could have a chance to speak. Finally, coworker help included the strategy to talk with coworkers about stressors and attitudes that may lead to potential bullying incidents. This idea was exemplified by the following participant remark: "Interrupt and give each nurse a chance to speak, valuing each opinion. Talk with each nurse regarding stressors and attitudes." The second relationship-level category was Addressing bully after event and focused on how to prevent repeated WB occurrences by speaking directly to the bully after the event. Some participants believed it was important to "confront the bully" and call out their negative behaviors. Specifically, participants wanted to let the bully know "how WB behavior makes one uncomfortable." Participants also felt it was important to prevent future WB by addressing the bully directly rather than going to management or human resources. This perspective was repeated by numerous respondents and exemplified by the following quote: "They could have spoken to the individual who made the comment instead of going straight to the managers." Overall, addressing the bully was viewed as a strategy to salvage one's future working relationship with the aggressor.

Ideas to prevent future bullying incidents that targeted the unit or work organization were categorized at the communitylevel and included two categories of ideas. Fostering positive, team-oriented work environment focused on unit level strategies to promote a workplace where WB would be less likely to occur. Ideas included promoting and creating a team focused work environment. Participants believe that future WB would be prevented if employees are focused on maintaining a cohesive team approach. For example, one participant wrote: "I think that when behavior like that [WB] occurs, peers that have been working on that unit for some time need to remind each other of the importance of team work and how that reflects on patient safety." In addition to a team focused environment, respondents also believed a supportive culture would prevent future WB. One participants' response to how to prevent future WB captured this sentiment: "Tell the experienced nurses that they were new once too and should be supporting new nurses rather than putting them down." The final idea suggested in this subdomain was to increase presence on the unit of a supervisor or manager. Having one's supervisor physically on the unit more often was viewed to foster a team-oriented attitude among nurses and potentially serve as a deterrent to future WB incidents.

The second category of the community-level strategies, Using resources to prevent $\mathrm{WB}$, focused on tapping into existing or creating new resources to prevent future bullying. Specific strategies identified included orienting preceptors of new nurses to their abilities so unrealistic expectations can be mitigated. One participant captured this idea succinctly: "Have the preceptor already informed of what the nurse's experience is before they begin working together so it is not a surprise." Another idea focused on the idea of providing mentorship programs so new nurses could develop relationships with others on the unit. Education of all staff, specifically on effective and professional communication, was viewed as an existing resource that could prevent future WB. "Communication about differences is always the key" and "establish and remind providers on handling discourse in a manner consistent of professionalism." Other strategies suggested at the community-level included adoption of a zero-tolerance pol- 
icy towards WB, have "management looking into the [WB] issue," and increase staffing on the unit so that there is less stress with work assignments.

\subsection{Stopping incidents of bullying behavior}

New nurse respondents who had directly experienced or witnessed behaviors of bullying reported use of various strategies to stop the incidents. The only individual-level strategy in this domain focused on Understanding with clarity the scope of practice and job description. A participant recalled how they used knowledge of their job description to quash bullying behavior incidents: "I intervened by standing up for myself. I told the bully that I know my job description, and I'm not going to do your job and mine too."

Four strategies used to stop bullying behavior occurred at the relationship-level. First, participants identified Discussing incident and/or seeking support from peers/manager as an approach when they experienced or witnessed bullying behavior (e.g. "I supported the peer after [the incident]", "I myself spoke to the charge nurse and explained the situation"). One participant even reported that this strategy was successful: "I went to inform my manager of the problem... Once he was told about the issue with the other staff nurse, the rudeness and bullying stopped."

Using de-escalation strategies was another approach the new nurses described to stop bullying behavior. De-escalation involves actions taken by the target to escape escalation of conflict or resolve conflict. Strategies ranged from getting everyone on the same page and adopting an appropriate tone of voice to reminding the aggressor you are still learning and trying to defuse the situation. One nurse recalled trying to de-escalate the situation by taking a positive spin: "I said thank you to both the nurses several times and stated that the incident helped my learning." Another nurse attempted to de-escalate by reminding the bully that they were both still learning: "I asked her how much experience she had in this position and when she said she had less than a year, I told her that we were both learning then because nursing requires lifelong learning."

A third relationship-level strategy to stop bullying behavior involved Upstanding activities. The term "upstanding" refers to proactive measures taken by people witnessing bullying behaviors taking place. Several of the respondents described someone stepping in to mediate the incident. For example, one nurse reported: "The nurse educator stepped in to help out, because she knows I was a new nurse and felt very overwhelmed at the moment." Another respondent reported a similar response by a co-worker: "The nurse preceptor pointed out to the [bully] that it wasn't okay to say

Published by Sciedu Press that and that I was in training." Another upstanding activity involved others encouraging the aggressor to understand others' perspectives. One nurse talked about how she intervened when she witnessed a bullying event: "I tried to get the nurse [bully] to see it from the travel nurse's point of view, which seemed to help. The next encounter went much better."

The fourth relationship-level strategy to stop bullying behavior included participants reports of Responding with aggression. Some participants stated that they tried to stop the incident by talking over the aggressor to redirect the conversation. One nurse recalled how she used this strategy: "I stopped the aggressor from talking over our peer and asked her [peer] to begin her report again." Others responded aggressively by calling out bullying behavior as being unacceptable. This strategy was illustrated by one new nurse's recollection of how she handled an incident of workplace bullying behavior:

I stopped report and stated 'I don't know what your deal is or what you are trying to accomplish by interrupting and making gestures. I am new to your unit and did the best I could with resources available. I apologize if that means you, as the seasoned nurse will have to clean up some of what I have left behind, but your attitude leaves a lot to be desired and I will appreciate you not constantly interrupting and making gestures.'

Community-level approaches used to stop bullying behavior consisted of two strategies. First was Re-establishing equilibrium where participants described efforts made by unit employees and leadership to help the work environment get back to normal. A few participants reported bullying behaviors were stopped by keeping the bully away from others (e.g., "My charge nurses just try to keep her [bully] away from everyone else. Quite a few nurses are hoping that she quits soon"). Others described how bullying behaviors were stopped and balance restored on the unit through adjustments to nurse/patient assignments "to be more fair" - potentially reducing a source of stress. Finally, bullying behaviors were stopped and equilibrium restored through the removal of or firing the bully from the unit. One participant recalled how the bullying finally ended when "the nurse manager made the bullying nurse leave the ED for another unit." The second community-level strategy to stop bullying behaviors was Engaging the system. Ideas described stopping bullying behaviors through use of existing resources available within the organizational system. For example, a few participants said they reported the bullying behavior incident using the workplace system available (e.g., safety report, incident report). 
One participant's response clearly articulated this approach: "After the incident we wrote a safety report and the charge nurse called the bully's supervisor directly." Others reported involving Human Resources (HR) and Management to get the bullying to stop. Finally, a few participants recalled how action was taken on the unit to investigate the alleged incident of bullying. One participant reported the following unit-level response to stop bullying behaviors: "The head of HR and the head of the department intervened. The manager was investigated and fired."

\subsection{Promoting others to act}

In the final domain, participants offered up ideas on what would need to happen or change in the workplace to promote people to action to stop WB when it occurs. All ideas were organized into one relationship- and two community-level strategies. The relationship-level strategy was Fostering relationships in the workplace. Ideas in this strategy focused on getting to know your coworkers better with the hope of identifying when they may be escalating toward WB. It also included a shared understanding of the role that other professions play in the healthcare environment. One new nurse expressed how addressing this issue could promote positive action.

It is very difficult as a nurse, new or not, to tell a doctor they are wrong or are not doing the right things for a patient. They are trained longer and have more experience than many of us. It would take a deeper understanding of both roles by each form of practice to be able to communicate better and not rush a conversation. There needs to be more respect for nurses who are up close and personal with patients and are watching them decline. MDs need to be especially attentive to those that say something along the lines of, 'Something is not right. Someone needs to see this patient.'

Finally, participants believe that better and more respectful communication between professions could help others address WB. For example, one participant postulated better communication could have been a strategy to use: "Next time [WB] happened, [I could] pull the physician aside respectfully and make sure she isn't taking offense to what is being said."

Two organizational-level strategies also identified ways to promote others to act against WB. One would be through Creating a safe work culture. Participants believed that safety needs to be a unit- and organizational priority to get employees to act and involves multiple ideas. First, creating a safe work culture would include making it safe to confront WB when it occurs. Participants currently express fear of becoming a WB target should they choose to intervene. One participant expressed feeling conflicted about not acting when they witnessed WB of a coworker: "I myself was still in orientation on the unit and felt too intimidated to stand up for the new nurse. I should have intervened anyway." Similarly, another participant stated: "I think being afraid of being picked on next made me not want to get involved." A second idea to create a safe work climate involved instituting policies about workplace behavior and clearly outlining expectations about professional interactions. Third, feeling safe to confront WB when it occurs requires institution of a supportive and respectful work environment for all. Participants specifically called for "doing classes on how to constructively correct someone" so that there would be "no fear of retaliation." Next, participants wanted to be assured that reporting WB will lead to a change. There is a belief that current inaction of nurses to address WB stems from the lack of action by leadership to address WB when it occurred in the past. Finally, a safe work climate also involves having safe and constructive outlets for work stress.

The second community-level strategy to promote others to act against WB focused on Preventing bullying through administrative support and resources. Ideas suggested included increased scheduling of staff on the unit to allay WB that may be related to overburdened staff members. Another idea was to have an additional level of command between management and staff. This idea was highlighted when a participant reported how inability to speak to her manager contributed to lack of action to address WB: "I would need to speak to my nurse manager who only works the weekdays. This incident happened on the weekend and I could not speak with her.' Finally, the last idea was to increase employee understanding and use of the existing reporting system for WB.

\section{Discussion}

Study findings indicate most newly-licensed nurse participants experience WB behavior. Of those participants, fewer than half had someone intervene. Strategies used to intervene represented individual, relationship, and community levels. Moreover, study findings suggest preventing future WB behavior or promoting intervening, multi-level strategies may be needed. On individual and relationship levels, interventions that support positive work environments and minimize incivil and bullying behaviors using the Social Ecological Model as a framework can mitigate the effects such behaviors. Mastroianni and Storberg-Walker (2014) postulate that individual level well-being is connected to organizational well-being in ways that are interdependent. ${ }^{[24]}$ 
Identifying how an individual assimilates into the social context of the workplace through activities designed to engender competence in one's workplace that emphasize leadership and engagement through collegiality are considerations to minimize the burden of incivil and bullying behaviors in the workplace. ${ }^{[24]}$ Creating opportunities for employees so that they look forward to coming to work, such as work balance initiatives, mindfulness, meditation spaces, and taking ownership over one's emotional intelligence, may be effective strategies to prevent WB behavior.

Strategies to better understand one's emotional intelligence such as Meyers-Briggs assessment and Strength Finders may provide opportunities for increased self-awareness. The Communications Style Inventory can facilitate knowledge of how one communicates with others. ${ }^{[25]}$ These self-awareness assessments may strengthen relationships among colleagues and mitigate the incidence and effects of bullying behaviors in the workplace. For example, men and women tend to have different communication styles. De Vries et al. (2011) found that women are more likely to become worrisome, tense, defensive, and project anger during stressful communications. ${ }^{[25]}$ In contrast, men generally use questioning techniques during communications perceived as argumentative and/or unsupportive. These differences in communication styles can lead to interpersonal conflict and contribute to perceptions that WB is or is not occurring. In a sample of undergraduate medical students, more men used questioning behaviors as a trait significantly more often than women and women used emotionality behaviors significantly more often than men. ${ }^{[26]}$ Respondents reported that getting to understand or know a coworker better (e.g., personality strengths, gender differences, communication styles) might provide recognition of escalation cues. It is unclear to what extent the impact of understanding one's attitudes and beliefs has on reducing workplace violence, but attitudes and beliefs are a component of many change theory models.

Strategies focused on the organizational level may hold the most promise for addressing WB in this population. Prevention of future bullying behavior starts with fostering a positive work environment where relationships between coworkers and other health professionals may lead to attitudes of mutual respect and collaboration. Prior research supports that creation of diverse, supportive work cultures helped reduce $\mathrm{WB}^{[4,11]}$ and nurses' experience of workplace incivility - a precursor to WB. ${ }^{[6,27]}$ Nurses who perceived leadership as supportive and unit staffing as adequate were less likely to experience incivility. ${ }^{[27]}$ In addition, organizational level training on effective and respectful communication skills can foster openness and mutual respect between and among health care professionals ${ }^{[28]}$ - important preventative strate- gies to decrease WB behavior. Therefore, organizations should consider implementing activities, such as monthly check-in meetings with managers and teambuilding with coworkers as well as communication skills training, to build rapport among all members of the healthcare team which in turn may moderate the effect of workplace incivility experiences on employee turnover. ${ }^{[6]}$

Another critical strategy to prevent future WB behavior involves increased awareness and usage of existing unit- and organizational-level resources to increase positive relationships and collaboration among staff members. Prior research supports the idea of positive relationships and collaboration as an important factor in addressing WB. Examining individual, work group, and organizational factors determined informal relationships and alliances between nurses in the organization may promote adoption of and support for bullying as normative behavior. ${ }^{[29]}$ However, lack of clarity and consistency in what constitutes WB behavior could be impeding the creation of healthy work environments. Johnson, Boutain, Tsai, and de Castro (2015) examined 22 documents from regulatory agencies and hospital organizations pertaining to WB or workplace violence. Results indicated a wide variety of terms (e.g., disruptive behavior, harassment, bullying, intimidation, verbal abuse, threatening behaviors, inappropriate behavior) were used across the guideline, regulatory, and policy and procedures documents examined. ${ }^{[30]}$ Moreover, most documents did not provide a clear definition of the term used. One way to create a positive work environment is through the development and adoption of organizational policies and education that use consistent terminology, define what behaviors constitute WB, and outline organizational-level sanctions of WB.

One distinct difference from prior research relates to the adoption of a zero tolerance for WB policy. Whereas prior research promotes adopting a zero-tolerance policy, ${ }^{[10-12]}$ our results suggest newly licensed nurses view a zero-tolerance policy as just one component of a safe work culture which integrates multiple strategies to prevent and reduce bullying.

Mitigation of WB requires accessible and feasible strategies to be implemented in the moment and in the long term. Strategies mentioned by participants included keeping the perpetrator away through changing nurses' patient assignments or removing the perpetrator from the unit as well as engaging existing organizational system such as reporting WB to managers and Human Resources. However, for managers to be effective, organizations should ensure managers are aware of current organizational WB policy and their role in addressing WB. ${ }^{[31]}$

Societal-level strategies also are needed to address WB 
among newly licensed nurses. Though participants in this study did not specifically recommend societal-level strategies, societal factors play a role in shaping nurses' beliefs about bullying in the workplace. At present, no federal or state-level laws make workplace bullying illegal in the United States. ${ }^{[32]}$ This is in direct contrast with societal efforts to address bullying experienced by students in U.S. schools. All 50 states and most U.S. territories have enacted state laws and/or policy requiring school districts to address the issue of bullying among students. ${ }^{[33]}$ Though passage of laws to make WB illegal will not eliminate WB altogether, passage of legislation does show a societal level commitment to making the work environment one where individuals can feel safe.

Future research on WB prevention and intervention strategies needs to be conducted with health care professionals especially newly licensed nurses. Considering study participants expressed a desire for a variety of WB strategies, interventions targeting multiple levels of prevention and mitigation need to be developed and tested. Moreover, educational interventions should be developed to teach nursing students about WB behaviors, so they are better equipped as a newly licensed nurse to deal effectively with this issue.

\section{Limitations}

Several limitations impact the transferability of findings to similar populations. First, participants were graduates from one of three university-based nursing programs located in the Midwestern U.S. Therefore, WB behavior reported by participants may represent behaviors unique to this region and not reflect WB behaviors experienced by newly-licensed nurses throughout the U.S. or in other countries. Second, convenience sampling strategy generated the small sample and therefore is biased. Convenience sampling methods resulted in a $42 \%$ response rate for this survey. However, it remains unknown if individuals who received but did not take the survey experienced WB behaviors as a newly licensed nurse and if so, was their WB behavior experience like those who did respond. Third, our convenience sampling strategy also resulted in an overrepresentation of participants who work in an intensive-care or critical care setting. Prior research indicates nurses working in intensive care units may experience higher levels of WB than non-intensive care nurses. ${ }^{[34]}$ Therefore, the results not be transferable to all newly licensed nurses. Finally, the cross-sectional survey study design prevented the research team from asking the participants follow-up questions or clarifying their responses. Therefore, researchers could only use the responses provided to the three open-ended questions in their qualitative data analysis.

\section{Conclusions}

The purpose of the study was to explore strategies to prevent and intervene during WB experienced by newly licensed nurses. Results indicate most participants experienced WB and less than half had someone intervene. Participants' responses included a variety of strategies to prevent and stop WB with most aimed at the organizational-level. Organizations that hire newly licensed nurses should evaluate current policies, procedures, and resources to identify opportunities to make improvements which could prevent future WB. Additional research is needed to develop and test strategies to prevent and mitigate WB in health care professionals especially those new to the professional environment.

\section{ACKNOWLEDGEMENTS}

This research study was funded by contract No. 211-2016M-90432 from the Centers for Disease Control and Prevention-National Institute for Occupational Safety and Health (CDC-NIOSH). The findings and conclusions in this report are those of the authors and do not necessarily represent the views of the National Institute for Occupational Safety and Health.

\section{Conflicts of InTEREST Disclosure}

The authors declare no conflicts of interest.

\section{REFERENCES}

[1] Berry PA, Gillespie GL, Fisher BS, et al. Psychological distress and workplace bullying among registered nurses. Online J Issues Nurs. 2016 Aug 10; 21(3): 8.

[2] Berry PA, Gillespie GL, Gates D, et al. Novice nurse productivity following workplace bullying. J Nurs Scholarsh. 2012 Mar; 44(1): 80-7. PMid:22339938 https ://doi .org/10.1111/j.1547-506 9.2011.01436.x

[3] Tong M, Schwendimann R, Zúñiga F. Mobbing among care workers in nursing homes: a cross-sectional secondary analysis of the Swiss
Nursing Homes Human Resources Project. Int J Nurs Stud. 2017 Jan; 66: 72-81. PMid:28017895 https ://doi.org/10.1016/j.ijnu rstu.2016.12.005

[4] Sliter M, Boyd E, Sinclair R, et al. Inching toward inclusiveness: diversity climate, interpersonal conflict and well-being in women nurses. Sex Roles. 2014; 71(1-2): 43-54. https://doi .org/10.1 007/s11199-013-0337-5

[5] Hurley J, Hutchinson M, Bradbury J, et al. Nexus between preventive policy inadequacies, workplace bullying, and mental health: qualitative findings from the experiences of Australian public sector employ- 
ees. Int J Ment Health Nurs. 2016 Feb; 25(1): 12-8. PMid:26752457 https://doi.org/10.1111/inm.12190

[6] Sguera F, Bagozzi RP, Huy QN, et al. Curtailing the harmful effects of workplace incivility: the role of structural demands and organizationprovided resources. J Vocat Behev. 2016 Aug-Oct; 95-96: 115-27. https://doi.org/10.1016/j.jvb.2016.08.004

[7] Ovayolu O, Ovayolu N, Karadag G. Workplace bullying in nursing. Workplace Health Saf. 2014 Aug; 62(9): 370-374. PMid:25102477 https://doi.org/10.3928/21650799-20140804-04

[8] Johnson SL. Workplace bullying in the nursing profession.1st .ed. USA : Singapore: Springer; 2018. https://doi .org/10.1007/ 978-981-10-5154-8_14-1

[9] Lee YJ, Bernstein K, Lee M, et al. Bullying in the nursing workplace: applying evidence using a conceptual framework. Nurs Econ. 2014 Sep-Oct; 32(5): 255-67.

[10] Ceravolo DJ, Schwartz DG, Foltz-Ramos KM, et al. Strengthening communication to overcome lateral violence. J Nurs Manag. 2012 Jul; 20(5): 599-606. PMid:22823215 https ://doi .org/10.111 $1 / \mathrm{j} .1365-2834.2012 .01402 . \mathrm{x}$

[11] Hoffman RL, Chunta K. Workplace incivility: promoting zero tolerance in nursing. J Radiol Nurs. 2015 Dec; 34(4): 222-7. https: //doi.org/10.1016/j.jradnu.2015.09.004

[12] Parker KM, Harrington A, Smith CM, et al. Creating a nurse-led culture to minimize horizontal violence in the acute care setting: a multi-interventional approach. J Nurses Prof Dev. 2016 Mar-Apr; 32(2): 56-63. PMid:26985749 https://doi .org/10 .1097/NND . 0000000000000224

[13] Castronovo MA, Pullizzi A, Evans S. Nurse bullying: a review and a proposed solution. Nurs Outlook. 2016 May-Jun; 64(3): 208-14. PMid:26732552 https://doi.org/10.1016/j.outlook. 2015 .11 .008

[14] Koh WMS. Management of work place bullying in hospital: a review of the use of cognitive rehearsal as an alternative management strategy. Int J Nurs Sci. 2016 Jun; 3(2): 213-22. https: //doi.org/10.1016/j.ijnss.2016.04.010

[15] Skehan J. Nursing leaders: strategies for eradicating bullying in the workforce. Nurse Leader. 2015 Apr; 13(2): 60-2. https ://doi .or $\mathrm{g} / 10.1016 / \mathrm{j} . \mathrm{mnl} .2014 .07 .015$

[16] D'Ambra AM, Andrews DR. Incivility, retention and new graduate nurses: an integrated review of the literature. J Nurs Manag. 2014 Sep; 22(6): 735-42. PMid:23927565 https://doi.org/10.111 $1 /$ jonm. 12060

[17] Grossman VGA. Hot Topics: teamwork essentials: success in the radiology department. J Radiol Nurs. 2013 Sep; 32(3): 139-40. https://doi.org/10.1016/j.jradnu. 2013.03.002

[18] Dahlberg LL, Krug EG. World report on violence and health. Geneva: World Health Organization (Switzerland); 2002. 1-21p.

[19] Violence prevention: The Social-Ecological Model: A framework for prevention [Internet]. Centers for Disease Control and Prevention (US); [updated 2018, Feb 20; cited 2019, May 6]. Available from: https://www.cdc.gov/violenceprevention/publich ealthissue/social-ecologicalmodel.html

[20] Gillespie GL, Grubb PL, Brown K, et al. "Nurses eat their young": a novel bullying educational program for student nurses. J Nurs Educ
Pract. 2017 Jul; 7(7): 11-21. PMid:28781715 https://doi .org/ 10.5430/jnep.v7n7P11

[21] Sandelowski M. Rigor or rigor mortis: the problem of rigor in qualitative research revisited. Adv Nurs Sci. 1993 Dec; 16(2): 1-8. PMid:8311428 https://doi.org/10.1097/00012272-199 312000-00002

[22] Sandelowski M. Whatever happened to qualitative description? Res Nurs Health. 2000 Aug; 23(4): 334-40. https://doi.org/10.1 002/1098-240X (200008) 23:4<334: :AID-NUR9>3.0.CO; $2-G$

[23] Lincoln YS, Guba EG. Naturalistic inquiry. Newbury Park, CA: SAGE Publications; 1985.

[24] Mastroianni K, Storberg-Walker J. Do work relationships matter? Characteristics of workplace interactions that enhance or detract from employee perceptions of well-being and health behaviors. Health Psychol Behav Med. 2014 Jan 1; 2(1): 798-819. PMid:25750820 https://doi.org/10.1080/21642850.2014.933343

[25] de Vries RE, Bakker-Pieper A, Konings FE, et al. The communication styles inventory. Commun Research. 2011 Jun; 40(4): 506-32. https://doi.org/10.1177/0093650211413571

[26] Doyen B, Vlerick P, Maertens H, et al. Non-technical attributes and surgical experience: a cross-sectional study comparing communication styles and attitudes in surgical staff, trainees and applicants. Int J Surg. 2019 Mar; 63: 83-89. PMid:30769216 https: //doi.org/10.1016/j.ijsu.2019.02.002

[27] Smith JG, Morin KH, Lake ET. Association of the nurse work environment with nurse incivility in hospitals. J Nurs Manag. 2018 Mar; 26(2): 219-226. PMid:28990326 https : //doi .org/10.1111/jo $\mathrm{nm} .12537$

[28] Nørgaard B, Ammentorp J, Kofoed P, et al. Training improves intercollegial communication. Clini Teach. 2012 Jun; 9(3): 173-177. PMid:22587317 https://doi.org/10.1111/j.1743-498X.20 $11.00525 . \mathrm{x}$

[29] Hutchinson M, Vickers MH, Wilkes L, et al. A typology of bullying behaviours: the experiences of Australian nurses. J Clin Nurs. 2010 Aug; 29(15-16); 2319-2328. PMid:20659206 https : //doi.org/10.1111/j.1365-2702.2009.03160.x

[30] Johnson SL, Boutain DM, Tsai JH, et al. An investigation of organizational and regulatory discourses of workplace bullying. Workplace Health Saf. 2015 Oct; 63(10): 452-61. PMid:26223898 https : //doi.org/10.1177/2165079915593030

[31] Johnson SL, Boutain DM, Tsai JH, et al. Managerial and organizational discourses of workplace bullying. J Nurs Adm. 2015 Sep; 45(9): 457-61.

[32] Nagele-Piazza L. Workplace Bullying and Harassment: What's the Difference? [internet]. [updated 2018 March 28; cited 2019, May 6]. Available from: https://www.shrm.org/resourcesandtools /legal-and-compliance/state-and-local-updates/pag es/workplace-bullying. aspx

[33] Laws, policies \& regulations [internet]. Washington (D.C): Department of Health and Human Services; [updated 2018 Jan 7; cited 2019, May 6]. Available from: https://www. stopbullying.gov /laws/index.html

[34] Ganz FD, Levy H, Khalaila R, et al. Bullying and its prevention among intensive care nurses. J Nurs Scholarsh. 2015 nov; 47(6): 505511. PMid:26444570 https://doi .org/10.1111/jnu. 12167 